\title{
Is Intraoperative Confirmation of Malignancy During Pancreaticoduodenectomy Mandatory?
}

\author{
Pramod Kumar Mishra • Sundeep Singh Saluja • \\ Asit Arora $\cdot$ Raja Kalayarasan
}

Received: 1 April 2012 /Accepted: 12 November 2012 / Published online: 21 November 2012

(C) 2012 The Society for Surgery of the Alimentary Tract

\section{Dear Editor,}

We read with great interest the article by Garcea et al. 'Is Intraoperative Confirmation of Malignancy During Pancreaticoduodenectomy Mandatory?' published in the issue 16 of the Journal of Gastrointestinal Surgery 2012. This article is about a very important dilemma faced by surgeons. We appreciate the authors for addressing this difficult problem. However, we have few queries regarding which we seek clarification from the authors. Authors have analysed their experience of 128 cases of which 62 had intraoperative frozen biopsy while 66 underwent Whipple's pancreaticoduodenectomy with clinical suspicion of malignancy. It appears that there is some selection bias involved in categorizing these patients. Thirty-five percent $(21 / 62)$ patients in the frozen biopsy group turned out to be benign compared to the $13.6 \%(9 / 66)$ in no intraoperative histology group. We would like to know how these two categories were arrived at. Authors have also mentioned about crossover in their study. If that is the reason for the significant difference in the incidence of malignancy, then that would question the basis for the classification into two groups.

A second problem is about obtaining the frozen biopsy. In cases of chronic pancreatitis with head mass, biopsy may be easily obtainable though it may still miss the malignant focus. In cases of lower end cholangiocarcinoma or small focus of malignancy nearby, a frozen biopsy is very difficult. Authors have not given their method of obtaining these frozen biopsies.

Another aspect needs to be mentioned. Endoscopic ultrasound and guided FNAC as well as PET-CT may reduce the number of patients where this dilemma remains. ${ }^{1,2}$ We would like to know from authors the number of patients these modalities were used including the results.

We encounter two different subgroups of patients - one with the lower end cholangiocarcinoma where biopsy/

P. K. Mishra $\cdot$ S. S. Saluja $(\bowtie) \cdot$ A. Arora $\cdot$ R. Kalayarasan Department of Gastrointestinal Surgery, G B Pant Hospital and Maulana Azad Medical College, Jawaharlal Nehru Marg, New Delhi 110002, India

e-mail: sundeepsaluja@yahoo.co.in cytology has not been positive and the second group comprising patients having chronic pancreatitis with head mass. While frozen biopsy is possible in the latter category, it is mostly clinical guidance in the former. It seems that the two categories of patients presented by authors may be conforming broadly to this classification. Authors have left us with a mathematical projection and broadly concluded that any of the approaches would be equivalent. We believe that a frozen biopsy may be undertaken in patients of chronic pancreatitis with a head mass, while resection surgery should be undertaken for lower end CBD blocks if malignancy is suspected. Obtaining a positive biopsy is not mandatory, especially for centres which have low mortality and morbidity of Whipple's pancreaticoduodenectomy. ${ }^{3,4}$ On balance, a $30 \%$ chance of cure in truly malignant cases weighs heavier against $3 \%$ mortality in patients who ultimately turn out to be benign.

Yours sincerely,

Pramod Kumar Mishra, Sundeep Singh Saluja, Asit Arora and Raja Kalayarasan

Department of Gastrointestinal Surgery, G B Pant Hospital and Maulana Azad Medical College

\section{References}

1. Delbeke D, Martin WH. PET and PET/CT for pancreatic malignancies. Surg Oncol Clin N Am. 2010; 19:235-254.

2. Eloubeidi MA, Varadarajulu S, Desai S, Shirley R, Heslin MJ, Mehra M, Arnoletti JP, Eltoum I, Wilcox CM, Vickers SM. A prospective evaluation of an algorithm incorporating routine preoperative endoscopic ultrasound-guided fine needle aspiration in suspected pancreatic cancer. J Gastrointest Surg. 2007; 11:813-819.

3. Mishra PK, Saluja SS, Gupta M, Rajalingam R, Pattnaik P. Blumgart's technique of pancreaticojejunostomy: an appraisal. Dig Surg. 2011; 28:281-287.

4. Schmidt CM, Turrini O, Parikh P, House MG, Zyromski NJ, Nakeeb A, Howard TJ, Pitt HA, Lillemoe KD. Effect of hospital volume, surgeon experience, and surgeon volume on patient outcomes after pancreaticoduodenectomy: a single-institution experience. Arch Surg. 2010; 145:634-640. 